\title{
Towards Generic Traffic Change Detection in the Data Plane
}

\author{
Gonçalo Matos \\ goncalo.o.matos@tecnico.ulisboa.pt \\ INESC-ID, Instituto Superior Técnico, \\ Universidade de Lisboa \\ Lisbon, Portugal
}

\author{
Salvatore Signorello \\ ssignorello@ciencias.ulisboa.pt \\ LASIGE, Faculdade de Ciências, \\ Universidade de Lisboa \\ Lisbon, Portugal
}

\author{
Fernando M. V. Ramos \\ fvramos@tecnico.ulisboa.pt \\ INESC-ID, Instituto Superior Técnico, \\ Universidade de Lisboa \\ Lisbon, Portugal
}

\begin{abstract}
Identifying traffic changes accurately sits at the core of many network tasks, from congestion analysis to intrusion detection. Modern telemetry systems perform traffic change detection but restrict their detection to heavy-hitters, failing to identify relevant traffic changes, including microbursts or low-volume attacks. We present K-MELEON, an in-switch online change detection system that identifies heavy-changes - instead of changes amongst heavy-hitters only, a subtle but crucial difference. K-MELEON is a variant of the $\mathrm{k}$-ary sketch (a well-known heavy-change detector) that leverages programmable switches for detection. To overcome the batch-based design of the original k-ary, K-MELEON features a new stream-based design that matches the switch's pipelined computation model and fits its tight constraints. The preliminary evaluation of the current prototype shows the potential of K-MELEON in achieving the same level of accuracy for online detection as the offline k-ary.
\end{abstract}

\section{CCS CONCEPTS}

- Networks $\rightarrow$ Network monitoring; Programmable networks; In-network processing.

\section{KEYWORDS}

change detection, sketch, data plane, software-defined networks

\section{INTRODUCTION}

The ability to detect traffic changes fast and efficiently is a fundamental requirement of many network operation tasks. Ideally, a change detection mechanism would analyse all packets from every flow and maintain all flow-related information. Such finegrained approach does not scale well, incurs in very high overheads, and is effectively not tractable without dedicated hardware. The typical change detection mechanisms therefore either rely on coarse-grained counters, like those provided by SNMP, or are sampling-based. The former provide useful aggregate statistics to detect major problems, but give up information that is often necessary to detect relevant changes, which are left sunk inside the aggregated traffic. The latter, common solution deployed in practice today, consists in sampling packets (e.g., NetFlow). However, the processing and bandwidth overheads of sampling make it infeasible to sample network traffic at sufficiently high rates.

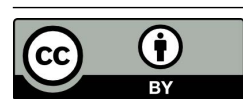

This work is licensed under a Creative Commons Attribution International 4.0 License.

CoNEXT-SW'21, December 7, 2021, Virtual Event, Germany

(c) 2021 Copyright held by the owner/author(s).

ACM ISBN 978-1-4503-9133-7/21/12.

https://doi.org/10.1145/3488658.3493784
An alternative to sampling that has been much explored recently is the use of sketches [3,9]. Proposed in the database community for summarizing data streaming datasets, sketches have interesting properties for the networking context too: space-efficiency and probabilistic memory-accuracy guarantees. Several sketch-based systems have been recently proposed, running different network monitoring tasks at line rate in commodity switches $[4,5,7,8]$. These modern systems are, however, restricted to change detection of heavy-hitters. The drawback is that, in practice, not all flows that experience significant changes are "heavy".

In this paper we present K-MELEON, a sketch-based system for generic change detection. Our starting point is the k-ary sketching algorithm [6], an efficient and accurate solution for the general change detection problem, but with some limitations that preclude its deployment. First, $k$-ary is an offline solution based on the analysis of bulk traffic traces. Network operators, however, need detection solutions that enable real-time decisions. Second, $k$-ary is softwarebased, limiting its performance, response time, and scalability. For instance, $\mathrm{k}$-ary is able to detect changes only in the order of minutes. As a result, it would fail to detect shorter yet relevant events in today's networks, including microbursts and short duration attacks.

K-MELEON leverages modern P4-programmable switch ASICs [1, 2] to implement a change detection mechanism that aims to be the online equivalent of k-ary, but that runs directly in the data plane. As a result, K-MELEON promises to achieve both generality and high performance.
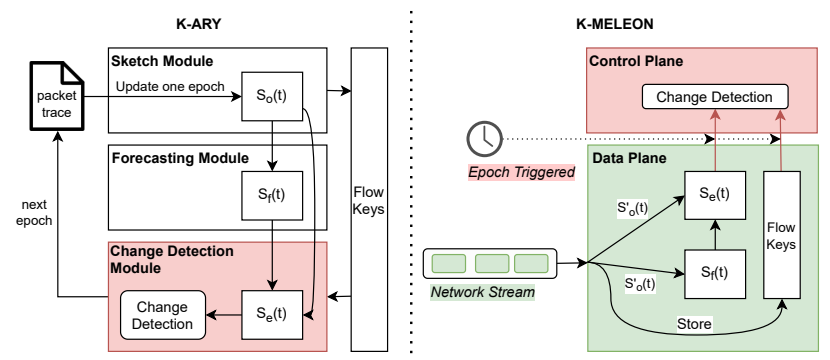

Figure 1: High-level block diagrams of k-ary and K-MELEON.

\section{SYSTEM DESIGN}

At a high level (Figure 1, left), the k-ary algorithm periodically computes an error $S_{e}(t)$ by comparing the forecasted values $S_{f}(t)$ based on past observations against the values currently observed $S_{o}(t)$ in the traffic stream. Running the logic of the k-ary in the data plane of $\mathrm{P} 4$-capable switches raises several challenges. First, the entire observed sketch data structure $S_{o}(t)$ must be traversed at once at the end of each epoch. P4, however, enforces limited packetby-packet memory accesses, not event-driven, concurrent accesses. Second, the arithmetic operations required by the forecasting model, 


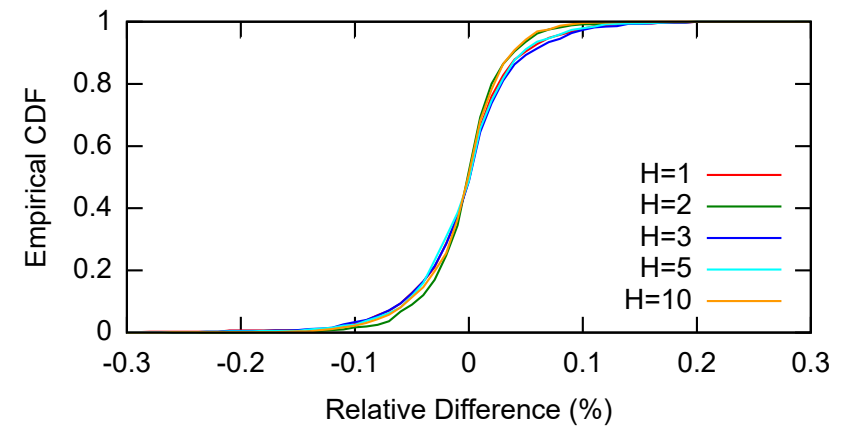

Figure 2: Empirical CDF values obtained for the relative difference between k-ary and K-MELEON for different sketch heights $(\mathbf{H})$.

and for the computation of the frequency moment estimates by the detection module, are rather complex, and $\mathrm{P} 4$ only allows for simple packet processing instructions. Both constraints are not a limitation of the language. They express restrictions that are common across high-throughput packet processing architectures to guarantee Tbps line rate processing.

A high level view of K-MELEON is also illustrated in Figure 1, sideby-side with the original k-ary algorithm. K-MELEON tackles the first problem by computing the forecast $S_{f}(t)$ and the error $S_{e}(t)$ sketches in a stream-based fashion. More specifically, K-MELEON modifies these operations, which were originally performed all at once at the end of each epoch, to compute them incrementally with each packet from the network stream. Part of the second problem stems from the Exponentially Weighted Moving Average (EWMA) forecasting model used in k-ary [6]. Applying EWMA, the computation of the forecast sketch in the current epoch is obtained as $S_{f}(t+1)=\alpha \cdot S_{o}(t)+(1-\alpha) \cdot S_{f}(t)$, thus requiring floatingpoint arithmetic $(\{\alpha \in \mathbb{R} \mid 0 \leq \alpha \leq 1\})$. K-MELEON converts the floating-point operations to multiplications via bit-shifts with the appropriate $\alpha$ values. The remaining operations include reading the error sketch and the flow-keys observed at the end of each epoch. In the current prototype these are performed by the change detection module that is offloaded to the control plane. We are currently working on moving these also to the data plane (Section 4).

\section{PRELIMINARY EVALUATION}

The goal of our preliminary evaluation is to understand whether K-MELEON preserves the detection accuracy of the k-ary algorithm, despite the approximations it incorporates. First, we validated the detection effectiveness of k-ary by extensively testing a Python implementation of the algorithm ${ }^{1}$ against several real traces containing labelled network attacks. Afterwards, we used the P4-reference software switch $b m v 2$ and tested our P4 prototype of K-MELEON against the same attack traces.

We computed the relative difference as defined in [6] to compare the detection achieved with K-MELEON and k-ary. Figure 2 shows that most of the mass is concentrated around the $0 \%$ point on the $x$-axis, with very few points that differ more than $0.2 \%$ from the corresponding k-ary ones. These experiments show K-MELEON achieves a similar level of accuracy as k-ary.

${ }^{1}$ We could not find an open-source implementation of the k-ary algorithm proposed in [6], so we have implemented it in Python and made it available at tinyurl.com/ctacawss.
While our prototype has been mainly tested on the $b m v 2$ software switch, the design of K-MELEON considers a high-speed switching ASIC as the main target platform. We have an early prototype for the Intel/Tofino switch [1] currently under test as part of our ongoing work.

\section{ONGOING \& FUTURE WORK}

Our initial evaluation of K-MELEON suggests that the proposed solution can perform change detection in the data plane of a P4capable switch, with small loss of accuracy when compared to the original k-ary algorithm. However, there are still some limitations:

Data Plane Detection. The current design of K-MELEON is still bottlenecked at the switch's CPU. At the end of each epoch, the control plane must read the entire error sketch and the keys stored in the current epoch to perform the detection. Our investigation led us to believe it is possible to overcome this challenge. We are currently exploring solutions that further leverage the processing ability of the data plane, coupled with specialized computing units available in the target switching platform.

Sketch Reversibility. Sketches can compress information about flows very effectively. However, these data structures typically do not preserve the flow keys. This is the case of both the original $\mathrm{k}$-ary and K-MELEON. As a result, these sketch data structures can detect changes but cannot readily recover the culprit flows ${ }^{2}$. We are exploring solutions that enable sketch reversibility by trading-off some memory efficiency.

Configuration space. Several parameters contribute to the detection accuracy of K-MELEON. We have not fully explored this configuration space. Rather, we have so far focused on finding configurations which on average worked well across the tested traces. However, our early experiments confirmed that the tuning of certain parameters may considerably help improve detection accuracy, so this is an avenue we will further explore.

\section{ACKNOWLEDGMENTS}

This work was partially supported by national funds through FCT via UIDB/50021/2020 and PTDC/CCI-INF/30340/2017 (uPVN) project.

\section{REFERENCES}

[1] [n. d.]. Intel Programmable Ethernet Switch Products. Retrieved April 22, 2021 from tinyurl.com/tofino-intel

[2] Pat Bosshart et al. 2013. Forwarding metamorphosis: Fast programmable matchaction processing in hardware for SDN. ACM SIGCOMM CCR 43, 4 (2013), 99-110.

[3] Graham Cormode et al. 2005. What's new: Finding significant differences in network data streams. IEEE/ACM ToN 13, 6 (2005), 1219-1232.

[4] Qun Huang et al. 2017. Sketchvisor: Robust network measurement for software packet processing. In Proc. of ACM SIGCOMM'17. 113-126.

[5] Qun Huang et al. 2018. Sketchlearn: relieving user burdens in approximate measurement with automated statistical inference. In Proc. of ACM SIGCOMM'18. 576-590.

[6] Balachander Krishnamurthy et al. 2003. Sketch-based change detection: Methods, evaluation, and applications. In Proc. of the 3rd ACM IMC. 234-247.

[7] Vibhaalakshmi Sivaraman et al. 2017. Heavy-hitter detection entirely in the data plane. In Proc. of ACM SOSR. 164-176.

[8] Tong Yang et al. 2018. Elastic sketch: Adaptive and fast network-wide measurements. In Proc. of ACM SIGCOMM'18. 561-575.

[9] Minlan Yu et al. 2013. Software Defined Traffic Measurement with OpenSketch. In 10th \{USENIX\} (\{NSDI\} 13) Symposium. 29-42.

\footnotetext{
${ }^{2}$ Unless by keeping all keys in memory, which would defeat the purpose of using memory-efficient sketches in the first place.
} 